\title{
Phenotypic plasticity in chemical defence allows butterflies to diversify host use strategies
}

\author{
Érika C. P. de Castro ${ }^{1}$, Jamie Musgrove ${ }^{2}$, Søren Bak ${ }^{3}$ W. Owen McMillan ${ }^{2}$, and Chris D. Jiggins ${ }^{3}$ \\ ${ }^{1}$ Butterfly Genetics Group, Department of Zoology, University of Cambridge, United Kingdom. \\ 2 Smithsonian Tropical Research Institute, Panama. \\ ${ }^{3}$ Plant Biochemistry, Department of Plant and Environmental Sciences, University of Copenhagen.
}

\begin{abstract}
Hostplant specialization is a major force driving ecological niche partitioning and diversification in insect herbivores. The cyanogenic defences of Passiflora plants keeps most herbivores at bay, but not larvae of Heliconius butterflies, which can both sequester and biosynthesize cyanogenic compounds. Here, we demonstrate that both Heliconius cydno chioneus, a host plant generalist, and $\mathrm{H}$. melpomene rosina, a specialist, have remarkable plasticity in their chemical defence. When feeding on Passiflora species with cyanogenic compounds they can readily sequester, both species downregulate the biosynthesis of these compounds. In contrast, when fed on Passiflora plants that do not contain cyanogenic glucosides that can be sequestered, both species increase biosynthesis. This biochemical plasticity comes at a significant fitness cost for specialist like $H$. m. rosina, as growth rates for this species negatively correlate with biosynthesis levels, but not for a generalist like $H$. c. chioneus. In exchange, $H . m$ rosina has increased performance when sequestration is possible as on its specialised hostplant. In summary, phenotypic plasticity in biochemical responses to different host plants offers these butterflies the ability to widen their range of potential host within the Passiflora genus, while maintaining their chemical defences.
\end{abstract}




\section{INTRODUCTION}

Phenotypic plasticity is widely recognised as an adaptation that allows organisms to survive in a variable environment [1]. Furthermore, there is interest in the idea that plasticity might permit populations to invade otherwise inaccessible niches or habitats [2][3]. Hostplant specialization is undoubtedly one of the most important forces driving diversification and shaping niche dimension for phytophagous insects [4][5]. Specialized insects often evolved not only to handle the chemical defences of their favorite hosts, but also to become dependent on plant compounds [6]. Whereas inducible defences of plants by herbivory has been well studied [7][8][9][10], there has been relatively little exploration of the mechanisms of biochemical plasticity in insect herbivores that could allow them to exploit diverse hosts [11].

The vast majority of aposematic butterflies acquired their toxic compounds from their larval hosts through sequestration. For example, the monarch butterfly sequesters cardenolides from milkweeds; swallowtails obtain Aristolochic acids from Aristolochiaceae; Ithomiini sequester pyrrolizidine alkaloids mostly from Solanaceae; and some toxic lycaenids acquired cycasin from Cycadales [6]. Sequestration of plant toxins during larval feeding is an adaptation that arose in many butterfly groups and plays an important role in the antagonist coevolution with their hosts. In contrast to most butterflies, Heliconius species have the ability to both sequester and synthesise their own chemical defences. All Heliconius butterflies can de novo biosynthesize aliphatic cyanogenic glucosides (CNglcs) using the amino acids valine and isoleucine as precursors [12] (see Figure 1 for CNglcs structures). Their Passiflora host plants are also chemically defended by a broad range of CNglcs [13], of which Heliconius can sequester aromatic, aliphatic, and especially simple cyclopentenyl during larval feeding [14][15][16]. To prevent sequestration, plants have responded by chemically modifying their defensive compounds. As an example, $H$. melpomene larvae can sequester cyclopentenyl CNglcs but cannot sequester sulfonated cyclopentenyl CNglcs from $P$. caerulea [15]. Other modified cyclopentenyl CNglcs, such the bis-glycosilated CNglcs, passibiflorin from $P$. biflora, have not yet been tested for sequestration. Disabling sequestration would not make these plants distasteful or toxic for Heliconius, but it could reduce their I value as a host and have deleterious effects on their fitness. From the perspective of the herbivores therefore, switching between biosynthesis and sequestration of toxins could allow butterflies to colonise a wider array of potential host plants independently of sequestration, while also maintaining their chemical defences. Here, we explore phenotypic plasticity in this trade-off in two Heliconius species with different host use strategies.

The closely related species Heliconius melpomene and Heliconius cydno (diverged 2 MYA) are often found in sympatry and their reproductive isolation is not complete (hybrid males are fertile) [17]. $H$. 
melpomene is widespread in tropical America and lays eggs on several Passiflora species, but where it co-occurs with Heliconius cydno, is an ecological specialist, ovipositing mainly on P. menispermifolia (Panama) or P. oerstedii (Costa Rica and Colombia), although larvae are able to feed on a variety of species. In contrast, H. cydno is more generalist and oviposits on many Passiflora species [18][19][20][21]. These differences in oviposition preferences are genetically controlled [21]. Broadly, larval mortality and growth of both species are similar on different hosts [22], but a field experiment showed slightly higher establishment probability for H. m. rosina on P. menispermifolia [21]. Overall, experiments to date show only weak evidence for any adaptive advantage to the host specialisation of $H$. melpomene as compared to the more generalist strategy of $H$. cydno.

Nonetheless, these species show different host use strategies and feed on a variety of host plants with different chemical composition. Here, we take advantage of this ecology to explore plasticity in the balance between sequestration and biosynthesis of cyanogenic compounds among these two butterfly species, fed on four Passiflora species that produce different CNglcs (Table 1). We also examine growth rates to explore whether there are possible trade-offs in fitness when feeding on different host plants or adopting different strategies of chemical defence. Phenotypic plasticity in sequestration versus biosynthesis of $\mathrm{CNglcs}$ defences could facilitate host switching and diversification of Heliconius across the Passiflora radiation.

Table 1. The CNglcs composition of the Passiflora species utilized in this study.

\begin{tabular}{|c|c|c|c|}
\hline & & \multirow{2}{*}{\multicolumn{2}{|c|}{ Modified }} \\
\hline \multirow{2}{*}{$\begin{array}{l}\text { Aliphatic } \\
\text { CNglcs }\end{array}$} & Aromatic & & \\
\hline & & CNglcs & CNglcs \\
\hline
\end{tabular}

\begin{tabular}{lllll}
\hline P. vitifolia & - & - & - & $\begin{array}{l}\text { Tetraphyllin B } \\
\text { sulphate }\end{array}$ \\
\hline P. platyloba & - & Prunasin & - & - \\
\hline P. menispermifolia & - & - & Deidaclin & - \\
P. biflora & - & - & - & Passibiforin
\end{tabular}

\section{METHODS}

\section{Butterfly rearing}

Butterflies used in this study were reared at the Smithsonian Tropical Research Institute, Gamboa, Panama. Mated female stocks of $H$. cydno chioneus and $H$. melpomene rosina were maintained in insectary cages and fed ad libidum with flowers (Psiguria triphylla, Gurania eriantha, Psychotria poeppigiana, Lantana sp.) and artificial nectar (10\% sugar solution). Plants of one of the four species 
61

used in the experiment - P. biflora, P. menispermifolia, P. platyloba, and P. vitifolia - were always kept in cages for oviposition. Eggs were collected daily from host plants and kept in closed plastic cups) until hatching. On the morning of hatching, larvae were transferred to treatment-specific cages onto individual shoots. Young shoots with no evidence of herbivory were selected to minimise the effects of variable host quality. Suitable shoots were sterilized and placed into water-filled bottles sealed with cotton. Cages were checked every day and fresh shoots provided regularly. Pupae were immediately removed, weighed after one day of pupation and taped on the lid of individual $350 \mathrm{ml}$ plastic tubes. After eclosion, butterflies were left in their individual tubes for a few hours to dry their wings and then removed for body measurements: total weight, forewing length and body length. Body length was measured from the end of the head to the end of the abdomen using mechanical callipers, and forewing length was measured from the central base to the most distal point. Butterflies were added into tubes containing $1.5 \mathrm{~mL}$ methanol $80 \%(\mathrm{v} / \mathrm{v})$, sealed with Parafilm and stored at $4{ }^{\circ} \mathrm{C}$.

\section{Chemical Analyses}

Samples were homogenized in 1.5 methanol $80 \%(\mathrm{v} / \mathrm{v})$ where they were soaked and centrifuged at $10,000 \times \mathrm{g}$ for $5 \mathrm{~min}$. Supernatants were collected and kept in HPLC vials at $-20^{\circ} \mathrm{C}$. Sample aliquots were filtered (Anapore $0.45 \mu \mathrm{m}$, Whatman) to remove insoluble components and diluted 50X times (v/v) in ultrapure water and injected into an Agilent 1100 Series LC (Agilent Technologies, Germany) hyphenated to a Bruker HCT-Ultra ion trap mass spectrometer (Bruker Daltonics, Bremen, Germany).

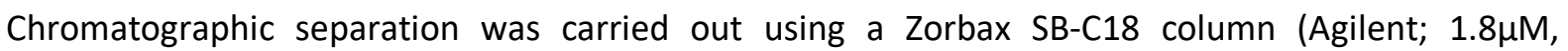
$2.1 \times 50 \mathrm{~mm})$. MS and LC conditions are described in [16]. The sensitivity of the analytical system was monitored by running a pooled sample after each 20 experimental sample.

Sodium adducts of CNglcs detected in the butterflies were identified by comparing their $\mathrm{m} / \mathrm{z}$ fragmentation patterns and RTs to authentic standards (Jaroszewski et al. 2002; Møller et al. 2016). Quantification of CNgIcs present were estimated based on the Extracted Ion Chromatogram peak areas of each compounds and calculated from a standard curve of linamarin, lotaustralin, and amygdalin.

\section{$\underline{\text { Statistical Analyses }}$}

All statistical analyses were performed using R version 3.5.1 (R Core Team, 2017). Two-ways ANOVA was used to examine the interaction between butterfly species, larval diet, and sex for different biological traits (pupal weight, adult weight, forewing length, body size, and total CNglcs). One-way ANOVA followed by Tukey HSD tests were used to make pairwise comparisons between diets and analyse, within butterfly species, the effects of each diet on the measured traits. 


\section{RESULTS}

Larval diet affected the CNglc composition in adult butterflies of $H$. melpomene and $H$. cydno (Figure 1). Both species sequestered deidaclin when fed as larvae on $P$. menispermifolia plants, although $H$. melpomene sequestered significantly more deidaclin than $H$. cydno (ANOVA, $F_{1,22}=8.851 ; p=0.00699$ ). Sequestration of deidaclin from $P$. menispermifolia was associated with a reduction of linamarin and lotaustralin biosynthesis in comparison with other diets. The modified CNglc passibiflorin from $P$. biflora and tetraphyllin B-sulphate from $P$. vitifolia were not found in both butterfly species raised on these diets, suggesting that they cannot sequester these compounds. Surprisingly, prunasin recently found in the haemolymph of larvae raised on P. platyloba [15] was not present in adults of either butterfly species. Instead, the derivative prunasin amide was found in adults reared on $P$. platyloba suggesting that they sequestered prunasin, but turned over into this compound to the corresponding amide during pupation.

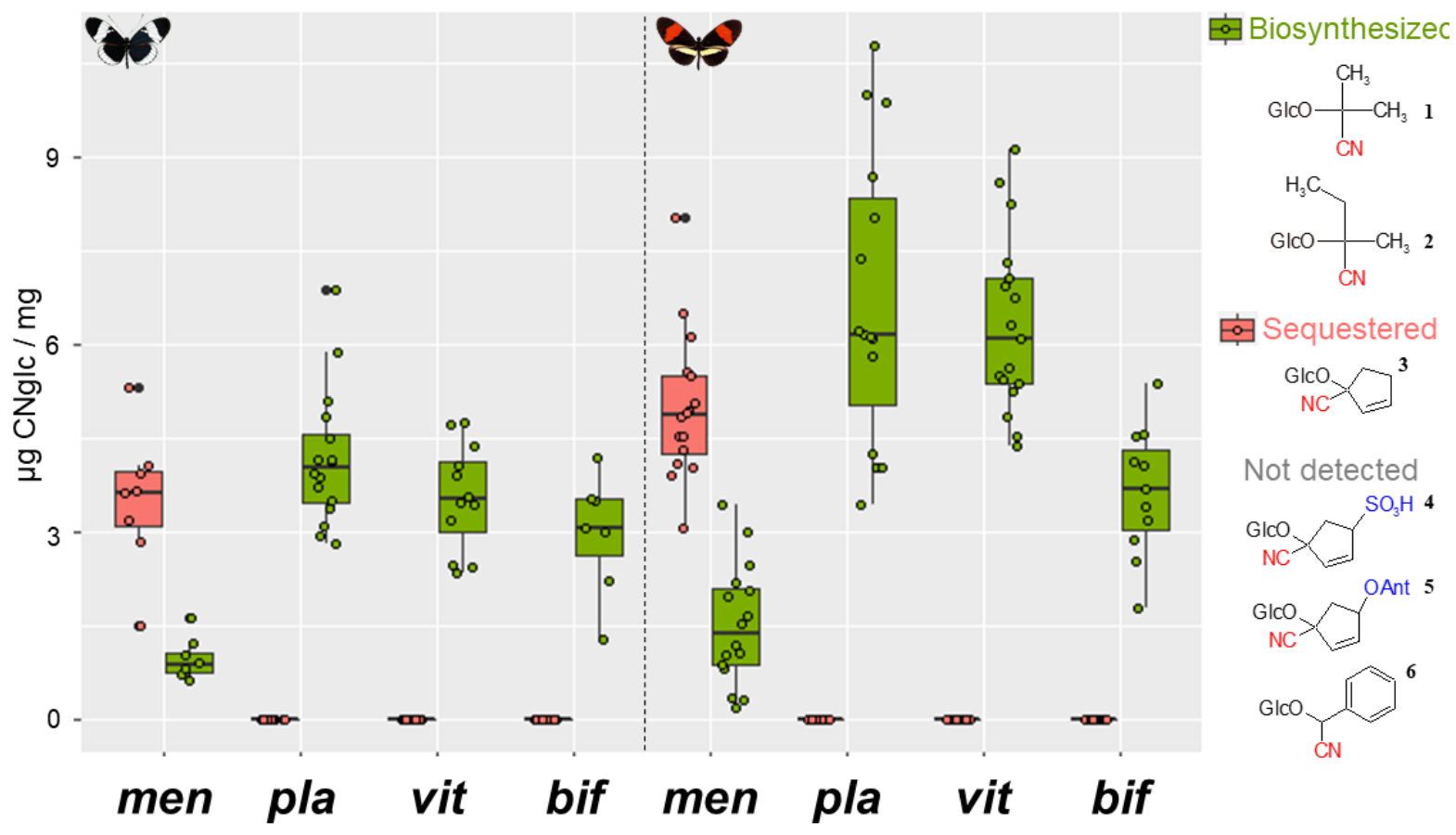

Figure 1. CNglc composition of $H$. cydno (left) and $H$. melpomene (right) raised on different Passiflora diet. Legend: vit $=P$. vitifolia, pla $=P$. platyloba, men $=P$. menispermifolia; bif $=P$. biflora. Green boxplots correspond to the biosynthesized cyanogens, linamarin ${ }^{1}$ and lotaustralin ${ }^{2}$, found in all butterflies. Salmon boxplots correspond to the sequestered CNglcs deidaclin ${ }^{3}$ only detected in butterflies raised on $P$. menispermifolia. Tetraphyllin B-sulphate ${ }^{4}$, passibiflorin ${ }^{5}$ and prunasin ${ }^{6}$ were not detected in butterflies, even thought they were present in the food plants $P$. vitifolia, $P$. biflora and $P$. platyloba, respectively. CNglcs present in each host plant is described in Table 1.

Larval diet not only influenced the composition, but also the total concentration of CNglcs in both species (ANOVA, H. cydno: $F_{3,39}=3.653, p=0.0205 ; H$. melpomene: $F_{3,55}=8.776, p=0.00007$ ) (Figure 2A). Both species had less CNglcs when reared on P. biflora, which they normally do not use as a host. 
117 On average, butterflies also had a higher CNglcs content when reared on P. menispermifolia than on

$118 P$. platyloba and $P$. vitifolia, though these differences were only statistically significant for $H$. cydno.

119 CNglc concentrations in $H$. cydno $(3.85 \pm 1.08)$ were on average lower than $H$. melpomene (5.96 \pm 120 1.97).

121 Larval diet also affected size and weight of both species. Forewing size of $H$. $c y d n o$ (ANOVA, $F_{3,39}=5.14$;

$122 p=0.004$ ) was larger and more strongly influenced by larval diet than $H$. melpomene (ANOVA, $F_{3,57}=$

$1234.0 ; p=0.012$ ) (Figure 2B). H. cydno had larger forewings when fed on P. vitifolia and P. biflora, and

124 smaller on $P$. menispermifolia and P. platyloba. In contrast, adults of $H$. melpomene had larger

125 forewings when reared on P. menispermifolia and P. biflora, and smaller on P. vitifolia and P. platyloba.

126 Sexual differences in forewing size were not observed in either species (ANOVA, $H$ melpomene: $F_{1,59}=$

$1270.369, p=0.546 ; H$. cydno: $\left.F_{1,41}=1.575, p=0.217\right)$. Broadly similar effects were seen for pupal weight,

128 butterfly weight and body size, as for forewing size (Figure S1).

In order to verify whether sequestration versus biosynthesis has a significant effect on fitness of both species, we tested for a correlation between concentration of biosynthesized CNgIcs and forewing size (Figure $2 \mathrm{C}$ ). In the generalist $H$. cydno, even though larval diet strongly affects forewing size, this effect is not correlated with whether they biosynthesize $\left(R^{2}=0.619, F_{1,41}=2.707, p=0.108\right)$ or sequester $\left(R^{2}=\right.$ $\left.0.09, F_{1,41}=4.081, p=0.05\right) \mathrm{CNg} \mid c s$. Whilst, in the specialist $H$. melpomene, there is a negative correlation between CNglc biosynthesis and forewing size $\left(R^{2}=0.1339, F_{1,57}=8.814, p=0.004\right)$, even when its favourite diet was removed of the analyses $\left(R^{2}=0.086, F_{1,53}=4.979, p=0.03\right)$. This suggests that $\mathrm{CNg}$ lcs biosynthesis has a fitness cost for $H$. melpomene rosina, which mostly lay eggs on $P$. menispermifolia and sequester CNglcs from it during larval feeding. Additionally, there was a positive correlation between forewing size and concentration of sequestered CNglcs in $H$. melpomene $\left(\mathrm{R}^{2}=\right.$ $\left.0.1466, F_{1,57}=9.979, p=0.003\right)$, indicating that larvae that are better sequestering $\mathrm{CNg} \mid \mathrm{cs}$ tend to turn into bigger butterflies. 
A)

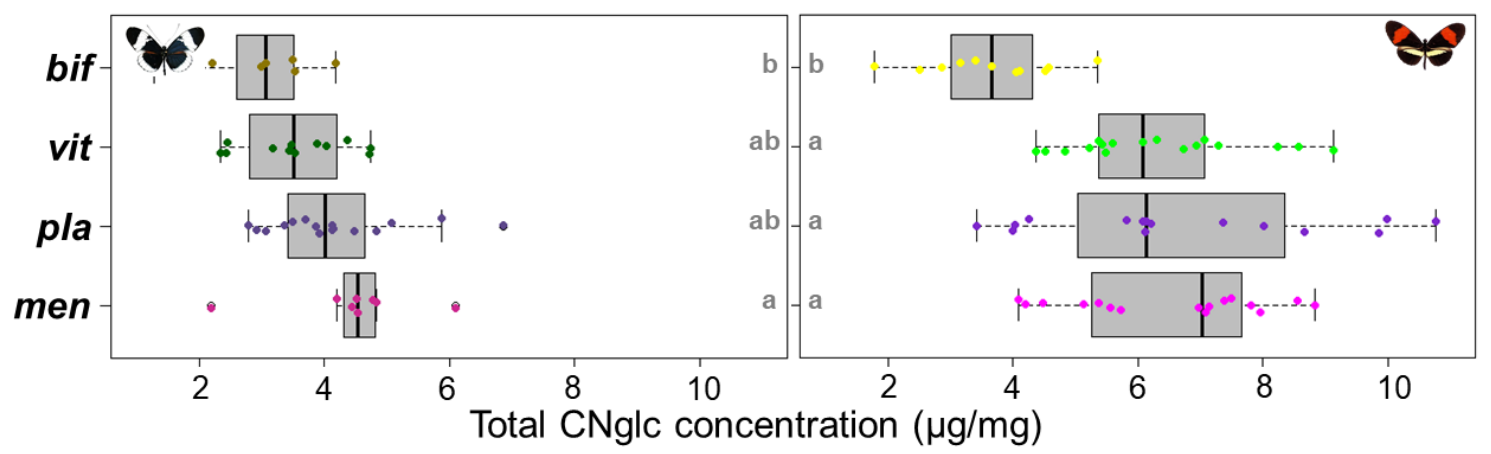

B)

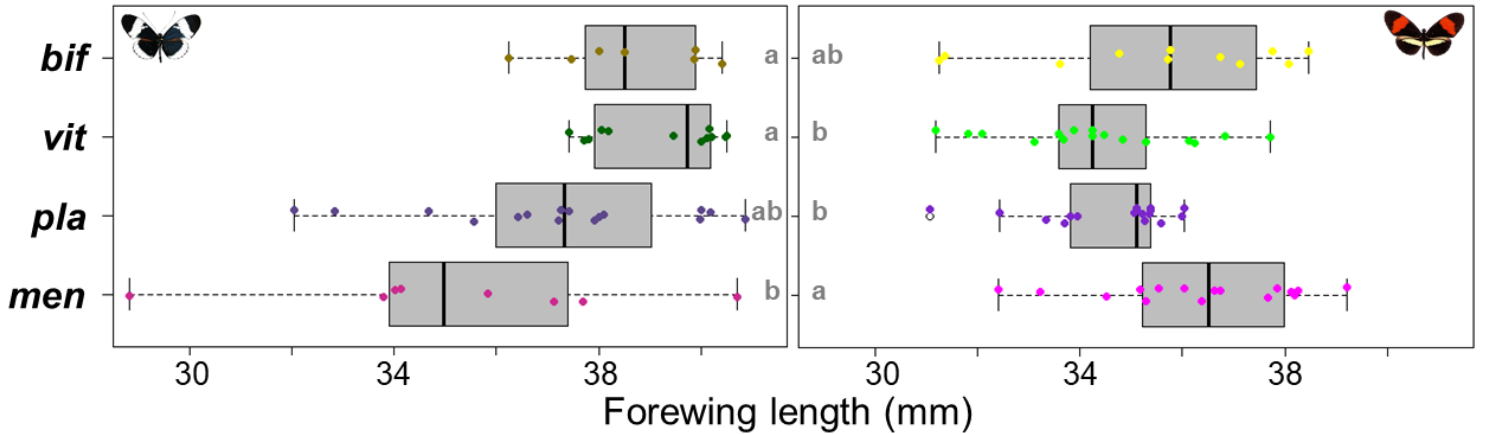

Forewing length $(\mathrm{mm})$

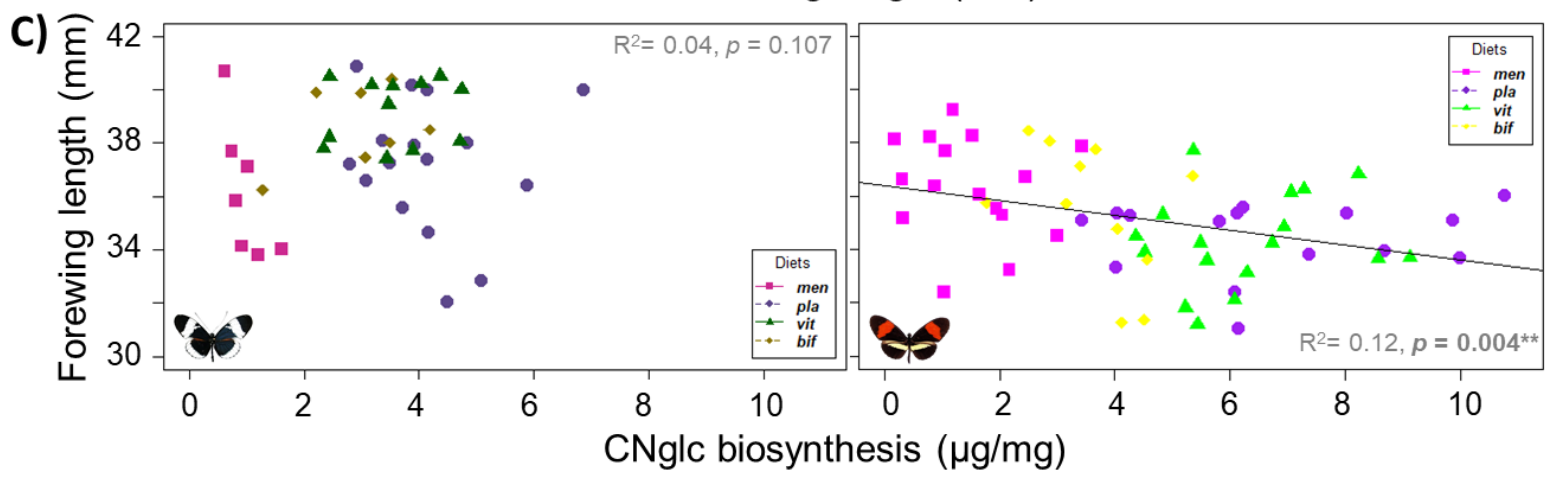

Figure 2. Effect of larval diet on $\mathbf{A}$ ) total $\mathrm{CNglcs}$ concentration and $\mathbf{B}$ ) forewing length of $H$. cydno (left) and $H$. melpomene (right). Letters over boxplots correspond to post-hoc comparisons (Tukey HSD) within butterfly species, where different letters indicate statistically significant treatments. C) Correlation between forewing length and concentration of biosynthesized CNglcs in $H$. cydno (left) and $H$. melpomene (right). Legend: vit $=P$. vitifolia, pla $=P$. platyloba, men= $P$. menispermifolia; $b i f=P$. biflora.

\section{DISCUSSION}

We documented, for the first time, plasticity in CNglc composition and concentration for both $\mathrm{H}$. melpomene rosina and $H$. cydno chioneus in response to their larval diet (Figure 1 and 2). We confirmed that when feeding on a plant with cyclopentenyl CNglcs that can be sequestered (i. e. deidaclin in P. menispermifolia), both butterfly larvae invest less in biosynthesis of aliphatic CNglcs, a trade-off that has previously been proposed at the level of inter-species comparisons [23][16]. This plasticity should facilitate Heliconius butterflies adapt to exploit different Passiflora hosts, they could utilize plants regardless of their CNglc profile because they can maintain their defences through biosynthesis when sequestration is not possible. 
Regardless of how they acquired their cyanogenic defences, both butterflies gained similar total concentration of $\mathrm{CNglcs}$ when raised on their natural host range ( $P$. platyloba, P. menispermifolia and P. vitifolia). A similar pattern has been observed in the six-pot burnet moth Zygaena filipendulae, another rare example of lepidopteran that can both de novo biosynthesize and sequester their chemical defences [25]. Z. filipendulae balance their cyanogenic content with biosynthesis in the absent of sequestration, however with deleterious consequences for their growth[26][27]. It is likely that, as in Zygaena moths, Heliconius have adaptations to optimize the energetic cost of their toxicity: deactivating the biosynthesis of CNglcs when these compounds are available for sequestration and reactivating it when they are not.

Although adult size and weight of $H$. cydno were strongly influenced by their larval diet (Figure 2), these differences were not correlated with whether they acquired their CNglcs through biosynthesis or sequestration. This suggests that plasticity in the generalist species does not come with a significant energetic cost. In contrast, $\mathrm{H}$. melpomene grows bigger (Figure $2 \mathrm{~B}$ and $\mathrm{S} 1$ ) when favouring sequestration over biosynthesis, suggesting that it has adapted to its specialist lifestyle and has a significant cost to the plasticity involved in switching host plants.

Smiley (1978) emphasized that ecological factors involved in the initial choice of a host plant might not be the same that led to the maintenance of this preference. It seems likely that the Panamanian $H$. melpomene only recently evolved a preference for $P$. menispermifolia. Once this oviposition preference established, selection for digestive adaptations to maximise the larval performance on this diet would take a place - e. $g$. increasing the efficiency of $\mathrm{CNglc}$ uptake from $P$. menispermifolia as we observed in this study (Figure 1). Local and recent adaptation to larval feeding on P. menispermifolia might also explain why H. melpomene performs only slightly better on this diet (Figure 2B and S1). Nonetheless, for the preferred host $P$. menispermifolia we have shown, for the first time, that this is a good host for $H$. melpomene, but a less optimal host for $H$. cydno.

In Panama, avoidance of interspecific competition is likely to be a major force shaping the evolution hostplant range, since coexistent Heliconius species rarely shared oviposition preference for the same Passiflora: H. erato lays eggs preferably on P. biflora, H. hecale on P. vitifolia, H. sara on P. auriculata and H. melpomene on P. menispermifolia[21][28]. Niche partitioning not only happens for Passiflora hosts, but also at microhabitat level: whereas most Heliconius species, including the comimics $\mathrm{H}$. melpomene and H. erato, are found in open secondary forest, $H$. cydno and $H$. sapho are typically present in the closed-canopy [29]. A similar pattern of resources partitioning (plant and microhabitat) occurs in Colombia [19]. Thus, interspecific competition might have led $H$. melpomene to evolve specialized oviposition preferences for $P$. menispermifolia and pushed $H$. cydno to inhabit forest where 
191

192

193

194

195

196

197

198

199

200

201

202

203

204

205

206

207

208

209

210

Passiflora species are less abundant and a generalist strategy might be favoured. The phenotypic plasticity in their biochemistry enabled Heliconius butterflies to widen their range of Passiflora host and led to niche diversification while maintaining their chemical defences, allowing the coexistence of multiple Heliconius species.

Finally, the vast majority of aposematic moths and butterflies sequester their toxic compounds from their larval host, emphasizing the importance of this process in the coevolution between plants and lepidopterans [30]. In turn, many Passiflora species seems to have modified their cyclopentenyl CNglcs to disable sequestration by heliconiines [16]. Here, we show that the two modified CNglcs passibiflorin (bis-glycosilated) and tetraphylli-B sulphate (sulphonated) were not sequestered by both Heliconius species, suggesting counter-evolution in the plants to deter their herbivores.

Our findings, based on Heliconius butterflies and its Passiflora host, highlight the importance of phenotypic plasticity in biochemical traits for the diversification of herbivorous insects. A large proportion of global biodiversity is represented by tropical herbivorous insects, so understanding how genetic and plastic traits allow species to adapt their host niche and permit species to coexist is an important step towards understanding biodiversity.

\section{REFERENCES}

1. Ashander J, Chevin LM, Baskett ML. 2016 Predicting evolutionary rescue via evolving plasticity in stochastic environments. Proc. R. Soc. B Biol. Sci. 283. (doi:10.1098/rspb.2016.1690)

2. Fordyce JA, Nice CC. 2008 Antagonistic, stage-specific selection on defensive chemical sequestration in a toxic butterfly. Evolution (N. Y). 62, 1610-1617. (doi:10.1111/j.1558$5646.2008 .00388 . x)$

3. Turner KG, Fréville H, Rieseberg LH. 2015 Adaptive plasticity and niche expansion in an invasive thistle. Ecol. Evol. 5, 3183-3197. (doi:10.1002/ece3.1599)

4. Ehrlich PR\&, Raven RJ. 1964 Butterflies and Plants : A Study in Coevolution Author. Soc. Study Evol. 18, 586-608.

5. Althoff DM, Segraves KA, Johnson MTJ. 2014 Testing for coevolutionary diversification: Linking pattern with process. Trends Ecol. Evol. 29, 82-89. (doi:10.1016/j.tree.2013.11.003)

6. Nishida R. 2002 Sequestration of defensive substances from plants by Lepidoptera. Annu. Rev. Entomol. , 57-92. 
221

7. Gleadow RM, Møller BL. 2014 Cyanogenic glycosides: synthesis, physiology, and phenotypic plasticity. Annu. Rev. Plant Biol. 65, 155-85. (doi:10.1146/annurev-arplant-050213-040027)

8. Agrawal AA, Conner JK, Johnson MTJ, Wallsgrove R. 2002 Ecological genetics of an induced plant defense against herbivores: Additive genetic variance and costs of phenotypic plasticity. Evolution (N. Y). 56, 2206-2213. (doi:10.1111/j.0014-3820.2002.tb00145.x)

9. Metlen KL, Aschehoug ET, Callaway RM. 2009 Plant behavioural ecology: Dynamic plasticity in secondary metabolites. Plant, Cell Environ. 32, 641-653. (doi:10.1111/j.13653040.2008.01910.x)

10. Baldwin IT. 1999 Inducible nicotine production in native Nicotiana as an example of adaptive phenotypic plasticity. J. Chem. Ecol. 25, 3-30. (doi:10.1023/A:1020880931488)

11. Petschenka G, Agrawal AA. 2015 Milkweed butterfly resistance to plant toxins is linked to sequestration, not coping with a toxic diet. Proc. R. Soc. B Biol. Sci. 282.

(doi:10.1098/rspb.2015.1865)

12. Nahrstedt A, Davis RH. 1983 Occurrence, variation and biosynthesis of the cyanogenic glucosides linamarin and lotaustralin in species of the Heliconiini (Insecta: Lepidoptera). Comp. Biochem. Physiol. -- Part B Biochem. 75, 65-73. (doi:10.1016/0305-0491(83)90041-X)

13. Jaroszewski JW, Olafsdottir ES, Wellendorph P, Christensen J, Franzyk H, Somanadhan B, Budnik B a, Jørgensen LB, Clausen V. 2002 Cyanohydrin glycosides of Passiflora: distribution pattern, a saturated cyclopentane derivative from P. guatemalensis, and formation of pseudocyanogenic alpha-hydroxyamides as isolation artefacts. Phytochemistry 59, 501-11.

14. Engler HS, Spencer KC, Gilbert LE. 2000 Preventing cyanide release from leaves. Nature 406, 144-5. (doi:10.1038/35018159)

15. Pinheiro de Castro ÉC, Demirtas R, Orteu A, Olsen CE, Motawie MS, Zikan Cardoso M, Zagrobelny M, Bak S. 2020 The dynamics of cyanide defences in the life cycle of an aposematic butterfly: Biosynthesis versus sequestration. Insect Biochem. Mol. Biol. 116, 103259. (doi:10.1016/j.ibmb.2019.103259)

16. de Castro ÉCP, Zagrobelny M, Zurano JP, Cardoso MZ, Feyereisen R, Bak S. 2019 Sequestration and biosynthesis of cyanogenic glucosides in passion vine butterflies and consequences for the diversification of their host plants. Ecol. Evol. 9, 5079-5093. (doi:10.1002/ece3.5062) 
251

17. Naisbit RE, Jiggins CD, Linares M, Salazar C, Mallet J. 2002 Hybrid sterility, Haldane's rule and speciation in Heliconius cydno and H. melpomene. Genetics 161, 1517-1526.

18. Smiley J. 1978 Plant Chemistry and the Evolution of Host Specificity: New Evidence from Heliconius and Passiflora. Science (80-. ). 201, 745-747.

19. Giraldo N, Salazar C, Jiggins CD, Bermingham E, Linares M. 2008 Two sisters in the same dress: Heliconius cryptic species. BMC Evol. Biol. 8. (doi:10.1186/1471-2148-8-324)

20. Naisbit RE. 2001 Ecological divergence and speciation in Heliconius cydno and H. melpomene. University of London. See https://www.ucl.ac.uk/taxome/lit/naisbitthesis02.pdf.

21. Merrill RM, Naisbit RE, Mallet J, Jiggins CD. 2013 Ecological and genetic factors influencing the transition between host-use strategies in sympatric Heliconius butterflies. J. Evol. Biol. 26, 1959-1967. (doi:10.1111/jeb.12194)

22. Smiley J. 1978 Plant chemistry and the evolution of host specificity: New evidence from Heliconius and Passiflora. Science (80-. ). 201, 745-747. (doi:10.1126/science.201.4357.745)

23. Engler-Chaouat HS, Gilbert LE. 2007 De novo synthesis vs. sequestration: Negatively correlated metabolic traits and the evolution of host plant specialization in cyanogenic butterflies. J. Chem. Ecol. 33, 25-42. (doi:10.1007/s10886-006-9207-8)

24. Engler-Chaouat HS, Gilbert LE. 2007 De novo synthesis vs. sequestration: negatively correlated metabolic traits and the evolution of host plant specialization in cyanogenic butterflies. J. Chem. Ecol. 33, 25-42. (doi:10.1007/s10886-006-9207-8)

25. Zagrobelny M, de Castro É, Møller B, Bak S. 2018 Cyanogenesis in Arthropods: From Chemical Warfare to Nuptial Gifts. Insects 9, 51. (doi:10.3390/insects9020051)

26. Zagrobelny M, Bak S, Ekstrøm CT, Olsen CE, Møller BL. 2007 The cyanogenic glucoside composition of Zygaena filipendulae (Lepidoptera: Zygaenidae) as effected by feeding on wild-type and transgenic lotus populations with variable cyanogenic glucoside profiles. Insect Biochem. Mol. Biol. 37, 10-8. (doi:10.1016/j.ibmb.2006.09.008)

27. Fürstenberg-hägg J, Zagrobelny M, Erik C, Jørgensen K, Lindberg B, Bak S. 2014 Transcriptional regulation of de novo biosynthesis of cyanogenic glucosides throughout the life-cycle of the burnet moth Zygaena fi lipendulae ( Lepidoptera ). Insect Biochem. Mol. Biol. 49, 80-89. (doi:10.1016/j.ibmb.2014.04.001) 
29. Estrada C, Jiggins CD. 2002 Patterns of pollen feeding and habitat preference among

30. Nishida R. 2002 Sequestration of defensive substances from plants by lepidoptera. Annu. Rev.
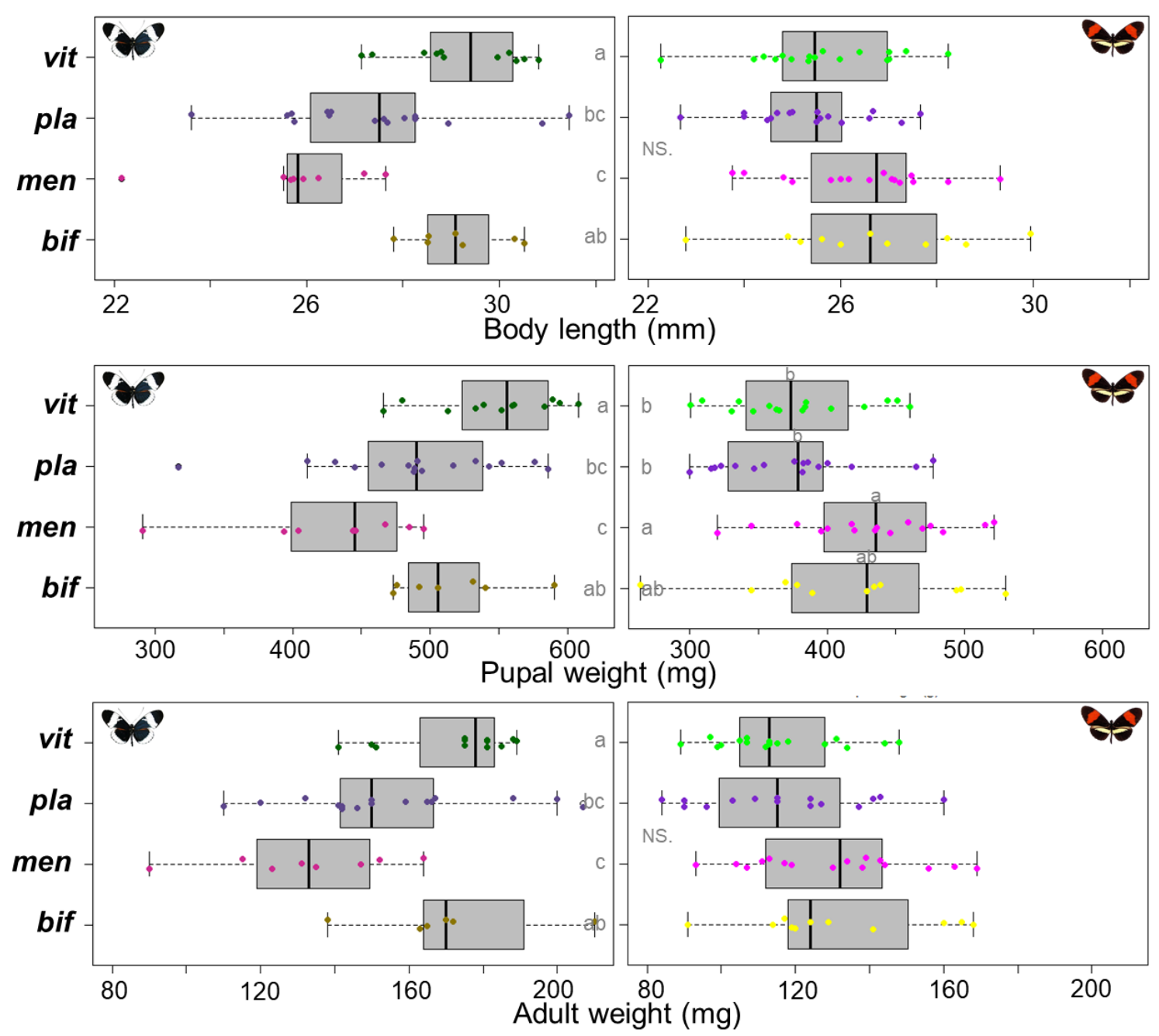

Figure S1. Effect of larval diet on body length, pupal weight and adult weight of $H$. cydno (left) and $H$. melpomene (right). Letters over boxplots correspond to post-hoc comparisons (Tukey HSD) within butterfly species, where different letters indicate statistically significant treatments. Legend: vit $=P$. vitifolia, pla= P. platyloba, men= P. menispermifolia; bif $=$ P. biflora. 
A)

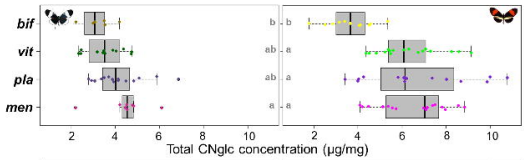

B)

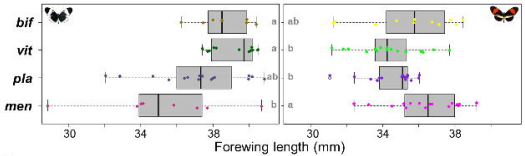

C

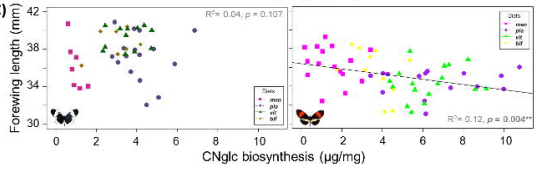

